\title{
Fast chopper for single radio-frequency quadrupole bunch selection for neutron time-of-flight capabilities
}

\author{
Asher Shor, ${ }^{1, *}$ Boaz Kaizer, ${ }^{1,3}$ Ilan Eliyahu, ${ }^{1}$ Tsviki Hirsh, ${ }^{1}$ Yossi Eisen, ${ }^{1}$ Arik Kreisel, ${ }^{1}$ \\ Leo Weissman, ${ }^{1}$ Amichay Perry, ${ }^{1}$ Israel Mardor, ${ }^{1,2}$ Shlomi Halfon, ${ }^{1}$ Eli Farber, ${ }^{3}$ \\ Gitai Feinberg, ${ }^{1}$ Daniel Kijel, ${ }^{1}$ Yonatan Mishnayot, ${ }^{1}$ Sergey Vaintraub, ${ }^{1}$ \\ Hila Yishai, ${ }^{1}$ Hodaya Dafna, ${ }^{1}$ and Shadi Yakobi $^{1}$ \\ ${ }^{1}$ Soreq Nuclear Research Center, Yavne 81800, Israel \\ ${ }^{2}$ School of Physics and Astronomy, Tel Aviv University, Tel Aviv 69978, Israel \\ ${ }^{3}$ Department of Electrical Engineering and Department of Physics, Ariel University, Ariel
}

(Received 13 September 2018; published 5 February 2019)

\begin{abstract}
A fast chopper system has been developed and tested for single bunch selection with the radio-frequency quadrupole (RFQ) accelerating element of the Soreq Applied Research Accelerator Facility (SARAF). The fast chopper consists of a high voltage (HV) deflector just before the RFQ, providing both positive and negative HV deflections and fast HV switching between polarities to enable momentary transmission of a single prebunch to the RFQ. Presently, the system enables single bunch selection for protons and deuterons at a repetition rate as determined by the user of up to $200 \mathrm{kHz}$, with bunch transmission of up to $50 \%$, and with neighboring bunch contamination of less than $15 \%$. Single bunch selection provides SARAF with fast neutron time-of-flight (TOF) capabilities. Measurements performed with liquid scintillation detectors show clear gamma and neutrons peaks, with TOF resolution of about 1 nanosecond FWHM. Beam dynamics simulations suggest possibilities for further improvements of the fast chopper and single bunch selection characteristics, with a significant lowering or elimination of the neighboring bunches, enhanced TOF resolution, and increased repetition rate to above $200 \mathrm{kHz}$. Fast neutron TOF capabilities, especially at phase II of SARAF, will provide exceptional opportunities for neutron induced reaction measurements for nuclear technology and fundamental research.
\end{abstract}

DOI: 10.1103/PhysRevAccelBeams.22.020403

\section{INTRODUCTION}

The use of beam choppers is prevalent in neutron facilities to provide for short temporal neutron pulses that enable neutron time-of-flight (TOF) measurements [1]. Various types of beam choppers and kickers have been developed for use with high current ion beams [2]. Development of "fast chopper" systems have also been pursued for adjusting the ion pulses to user's experimental requirements or for synchronizing ion pulses with subsequent acceleration components, high energy storage rings, etc. Sophisticated beam chopping systems are being developed for existing and planned high power proton drivers for next generation spallation sources and neutrino factories. Examples include the RAL-ESS chopping system [3], based in the medium energy beam transport (MEBT)

\footnotetext{
*Corresponding author. shor@soreq.gov.il

Published by the American Physical Society under the terms of the Creative Commons Attribution 4.0 International license. Further distribution of this work must maintain attribution to the author(s) and the published article's title, journal citation, and DOI.
}

section, with a slow-wave chopper consisting of several sets of deflecting electric fields and beam dumps operating in tandem to follow the velocity of the ion. A recent review of the ESS linac chopping system performance can be found in Ref. [4]. Further examples include the LANL and SNS chopping scheme [5] with slow traveling-wave structures based on a meander line and strip-coax helical structure to produce deflecting fields that follow the velocity of the ion. J-PARC [6] has a two-stage chopping system with a low energy beam transport (LEBT) prechopper based on energy modulation of the beam before entrance to the radio-frequency quadrupole (RFQ), and a horizontal kicker in the MEBT following the RFQ. The CERN Super Conducting Proton Linac [7] chopper consists of a double meander line. These structures require high voltage (HV) pulse rise and fall times of several nanoseconds to avoid partially deflected pulses that generate unwanted beam loss. Novel examples for high current include an $\mathrm{E} \times \mathrm{B}$ field chopper system described by Wiesner et al. [8], and a chopping system based on a new Einzel lens described by Leo et al. [9].

Fast chopping systems are also being developed for $\mathrm{rf}$ ion accelerators as "single bunch selectors." These bunching systems are designed to transmit only one rf period, taking 
advantage of the inherent bunching process of the rf accelerator that decreases the temporal width by a significant factor. Accelerator facilities that rely on a radio-frequency quadrupole (RFQ) preaccelerator buncher can especially gain from single bunch selection techniques, since the RFQ itself bunches each rf cycle of the beam to less than a tenth of the rf cycle. Several groups are developing single bunch selection capabilities with beam choppers based on electrostatic deflectors situated downstream of the RFQ and relying on traveling-wave pulses deflecting current structures based on meander lines sufficiently long to follow the desired RFQ bunch. Di Giacomo et al. [10] report on the development of a single bunch selection to be used at the Spiral II [11] accelerator complex planned for the Neutrons for Science (NFS) facility [12]. This technique relies on very fast HV pulses rise and fall time, approximately $5 \mathrm{~ns}$ or less, with the goal of no residual particles from the suppressed bunches [13]. A similar technique has been discussed by Madrack and Wildman [14] for single bunch selection following the 2.5 MeV $325 \mathrm{MHz}$ RFQ at Fermilab, which includes a pulse delaying microstrip structure for deflecting the beam, with high voltage $(1.2 \mathrm{kV})$ and fast (ns rise time) pulsers.

Techniques for fast chopping based on beam deflection before the entrance to the RFQ avoid the increased rigidity and dissipated power of the deflected beam. Nath, Stevens, and Wangler [15] discussed the possibility of single bunch selection by chopping the dc beam before the RFQ, emphasizing the low rigidity of the beam to be deflected and the low power required, but also highlighting the issues of space charge and temporal changes in beam neutralization, and the need to minimize emittance growth [15]. Emittance growth due to changes in beam neutralization was also addressed by Prost et al. [16], who suggest techniques for creating an unneutralized section in the chopper vicinity. Laxdal et al. [17] discussed a chopper system in the LEBT section of the ISAC accelerator complex comprised of two sets of deflection plates driven at two different $\mathrm{rf}$ frequencies, for proper subsequent matching of the beam exiting the RFQ to the postaccelerator section. About a decade ago, collaborators from the FNeuRad [18] project proposed pulsed fast neutron sources based on an electrostatic deflection by an rf HV resonator before the RFQ, a technique requiring high power and feasible for only high repetition rates due to the fast deflection that was required. Several years ago, Hamm et al. [19] reported on a single pulse subnanosecond proton RFQ based on a small $8 \mathrm{~mm}$ long electrostatic deflector before the RFQ, where the emphasis was on a technique to obtain a "single shot" RFQ bunch. The technique proved to be successful, but of limited repetition rate.

Recently, a fast chopper system for single RFQ bunch selection has been developed and tested at the Soreq Applied Research Accelerator Facility (SARAF) proton/ deuteron accelerator complex [20]. The fast chopper consists of electrostatic deflection plates positioned before the RFQ. The scheme for single bunch selection involves alternating between positive and negative deflection, where a fast $20 \mathrm{~ns}$ switch changes the deflection polarity and allows momentarily for a short enough burst to enter the RFQ to eventually become a single bunch. Techniques for single bunch selection are especially beneficial in the case of the SARAF RFQ, which operates at rf of $176 \mathrm{MHz}$ [21]. The RFQ takes $5.5 \mathrm{~ns}$ of dc beam and bunches it to $\Delta \mathrm{t}$ of approximately 0.3 nanoseconds, while accelerating the $20 \mathrm{keV} / \mathrm{u}$ beam to $1.3 \mathrm{MeV} / \mathrm{u}$ [22].

Single bunch selection with neutron pulses of less than 1 nanosecond make possible neutron TOF experiments of nuclear reactions that require ultrafast timing. Planned experiments include nuclear spectroscopy, study of fission fragments and prompt fission neutron spectra, and various aspects of nuclear astrophysics [23]. The inclusion of a fast chopping system for a variable energy proton/deuteron accelerators such as SARAF, with the ability to create quasimonochromatic neutron beams, can avoid the need for double time-of-flight measurements when measuring nuclear reactions where knowledge of both the incident and outgoing neutrons is required [24]. Fast neutron TOF techniques have also been developed for industrial applications, for example security applications, including luggage or container interrogation with neutron TOF to provide information on elemental composition of suspected items [25].

\section{SARAF PHASE I ACCELERATOR, LEBT, RFQ, AND SLOW CHOPPER SYSTEM}

The SARAF accelerator complex is designed for $40 \mathrm{MeV}$ proton/deuteron cw beams at $5 \mathrm{~mA}$ current, where the major usage is to provide high flux neutron beams for industrial and research applications [20]. Phase II will include a normal conducting "injector" system plus four cryomodules consisting of 27 superconducting half-wave resonator (HWR) accelerating elements [26]. Phase I of SARAF, with one prototype superconducting module (PSM) consisting of six HWRs, is currently operational and provides proton or deuteron beams of up to 4 and $5 \mathrm{MeV}$ respectively. Ongoing research projects of both applied and of academic nature have been conducted over the past several years [20].

The existing SARAF injector is foreseen as the dedicated injector system for phase II of the SARAF project. The SARAF injector consists of an electron cyclotron resonance (ECR) ion source, a low energy beam transport (LEBT), and a radio-frequency quadrupole accelerator buncher (RFQ). Figure 1 shows a schematic top view of the SARAF injector, including a slow chopper consisting of an electrostatic deflection plates and beam blocker. The slow chopper deflects the low energy beam at an angle of $20^{\circ}$ onto a water-cooled retractable beam blocker. A fast HV switch neutralizes the deflection HV for the required beam on-time. The slow chopper electronics provides for a minimum off-time of about $180 \mathrm{~ns}$, corresponding to a 


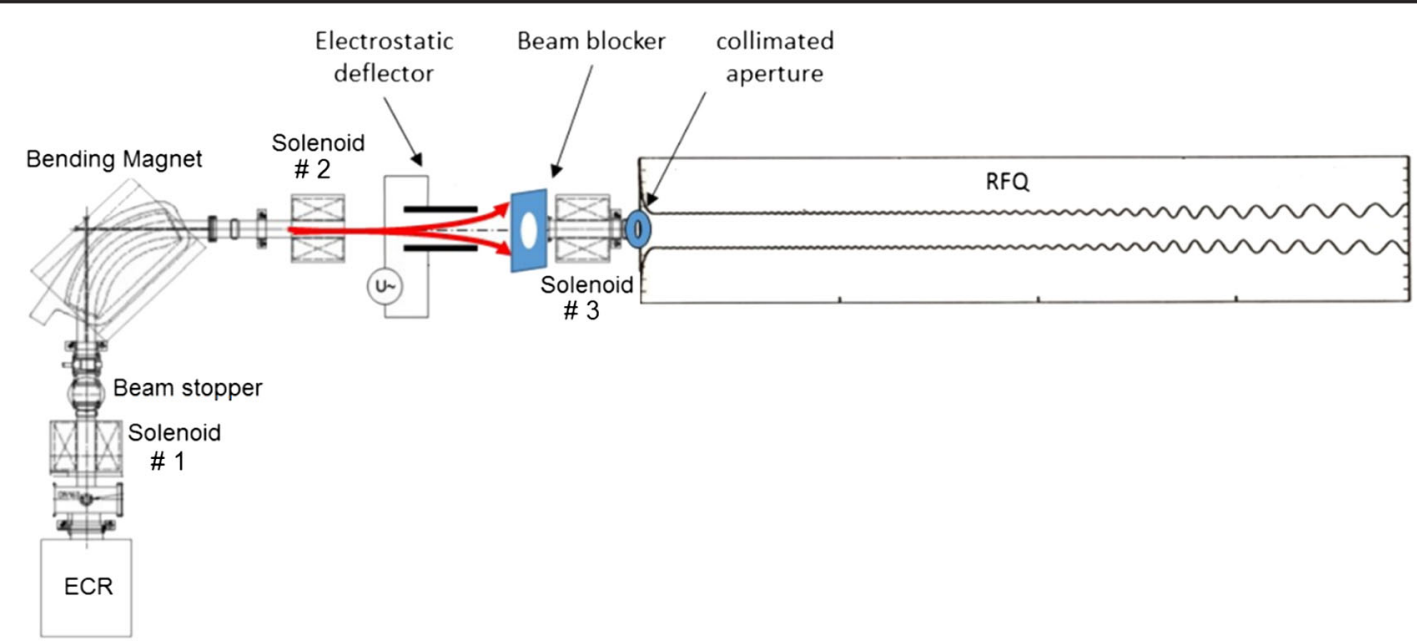

FIG. 1. Schematic top view of the SARAF injector, including the ECR ion source, LEBT, and RFQ, including deflection electrodes for beam-left and beam-right deflection and beam blocker.

transmitted pulse of $180 \mathrm{~ns}$, and at a maximum switching rate of up to $800 \mathrm{~Hz}$ [27]. The slow chopper deflection plates and electronics were developed at LNS Catania within the SPIRAL 2 program, and mounted at SARAF for tests and evaluation [28]. The main function of the slow chopper at SARAF and SPIRAL 2 is to provide a
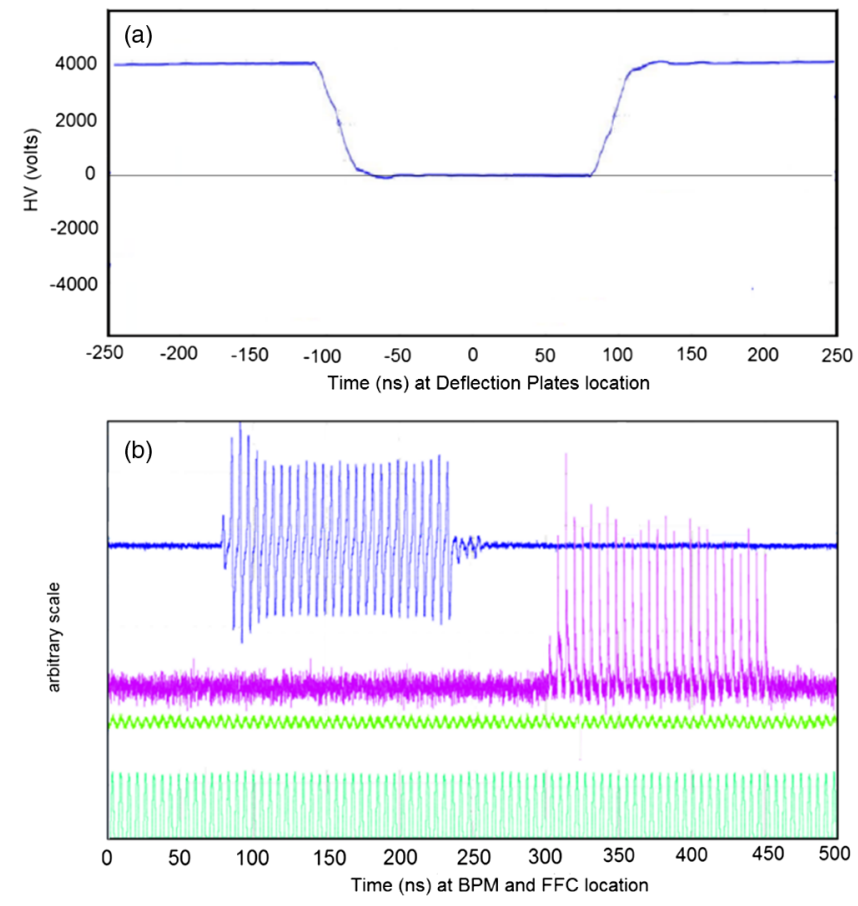

FIG. 2. Slow chopper operation: (a) HV on deflection platesconstant $\mathrm{HV}$ of $4 \mathrm{kV}$ for beam deflection with application of $180 \mathrm{~ns}$ pulse of reverse polarity for neutralizing the deflection field and allowing momentary transmitting of beam pulse. (b) Oscilloscope trace of beam induced signals on detectors. Green trace is main rf, blue trace is signal induced on the BPM situated in the MEBT, and purple trace is induced signal on the FFC situated 4 meters downstream. simple and effective mechanism for lowering beam current for beam tuning and diagnostic purposes and for ramping up of the beam duty cycle, without having to pulse the ion source or other essential components.

Figure 2 demonstrates the operation of the "slow chopper." A constant potential of $4 \mathrm{kV}$ is required for adequate deflection of protons. This is neutralized by a short HV pulse of opposite polarity applied when transmission of a beam pulse is desired. Figure 2(a) showing the HV profile on the deflection plates with a $180 \mathrm{~ns}$ neutralization pulse applied for beam transmission. Figure 2(b) shows the oscilloscope traces for the $180 \mathrm{~ns}$ transmitted beam pulse and bunching nature of the RFQ. The green trace shows the main accelerator $\mathrm{rf}$, the blue trace shows the induced pulses on the beam position monitor (BPM) positioned in the medium energy beam transport (MEBT) following the RFQ, and the purple trace shows the induced pulses on the fast Faraday cup (FFC) located in the diagnostic plate (D-plate), (following the PSM) about 6 meters downstream. It should be noted that a proton or deuteron at $20 \mathrm{keV} / \mathrm{u}$ in the LEBT section takes about $66 \mathrm{~ns}$ to traverse the $130 \mathrm{~mm}$ long deflection plates, corresponding to about $12 \mathrm{rf}$ periods. Thus, neutralizing the deflection $\mathrm{HV}$ for any short duration is not sufficient to transmit only one rf period to the RFQ.

\section{SIMULATIONS OF FAST CHOPPER AND CONDITIONS FOR SINGLE BUNCH SELECTION}

The initial idea for fast chopper stemmed from beam dynamics simulations that showed promising single bunch selection performance with the existing slow chopper deflection plates assuming alternating positive or negative HV deflection with fast switching [29]. The dc beam traverses the deflection plates and experiences constant transverse force while inside the field of the deflection plates. The section of the dc beam that passes midway 

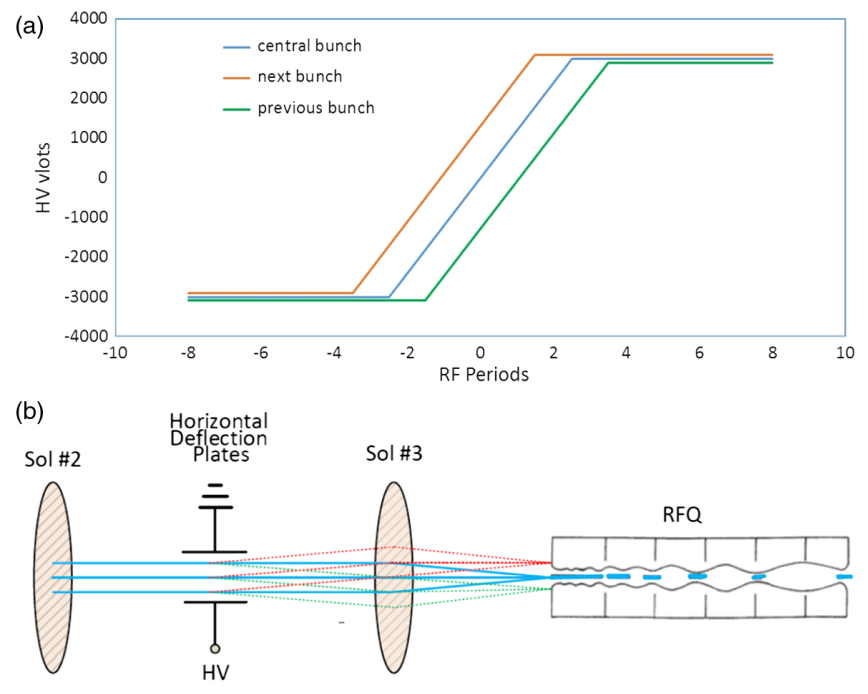

FIG. 3. Schematic diagram of the single bunch selection mechanism: (a) Deflection electric field felt by beam passing deflection plates for central bunch and "previous" and "next" bunches. (b) Diagram of undeflected and deflected beam focused onto RFQ, blue corresponding to central bunch, and green and red corresponding to previous and next bunches.

of the deflection plates when the polarity switch occurs experiences equal amounts of negative and positive deflection. The net transverse velocity gain is canceled out, and this section of the dc beam proceeds onward towards the RFQ to become the "central bunch." The section of the dc beam one rf cycle ahead experiences a net negative field for a time equal to two rf time periods, while the dc beam one rf cycle behind experiences a net positive deflection for a similar amount of time, both are deflected away from the RFQ acceptance, either to the beam right or to the beam left. The basic idea is represented schematically in Fig. 3(a). The length of time $\Delta \mathrm{t}_{\text {switch }}$ for the polarity switch is not crucial (we achieved $\Delta \mathrm{t}_{\text {switch }}$ of $28 \mathrm{~ns}$ or about five rf periods), it must be significantly less than the beam traversal time in the deflection plates (about $16 \mathrm{rf}$ periods including fringe field), and must be symmetric for the cancellation to take place effectively. The objective is for maximum transmission of the central bunch, and for neighboring bunches as low as possible.

A simple calculation illustrates the principle of the fast chopper single bunch selection. The beam between solenoid \#2 and solenoid \#3 is essentially a parallel beam [see Fig. 3(b)]. A net deflection caused by the deflection plates provides for a transverse velocity component. The angle of the beam emerging the deflection plates can be easily estimated. The longitudinal velocity is given by $v_{z}=\sqrt{\frac{2 q \phi}{m}}$, where $\mathrm{q}$ and $\mathrm{m}$ are the charge and mass of the proton/deuteron, and $\phi$ is the accelerating potential of the ion source. The transverse velocity as a result of the deflection, assuming a constant electric field $E$, is given by $v_{t}=\frac{q}{m} \frac{V}{d} \times \frac{\Delta L}{v_{z}}$, where $\boldsymbol{V}$ is the HV and $\boldsymbol{d}$ the separation of the deflection plates $(\boldsymbol{d}=92 \mathrm{~mm})$, and $\Delta \boldsymbol{L}$ is the net distance traversed in the deflection field that is not canceled out by opposite deflection. The deflection angle $\theta_{\text {def }}$ is given by

$$
\theta_{\mathrm{def}}=\frac{v_{t}}{v_{z}}=\frac{\Delta L}{2 d} \times \frac{V}{\phi}
$$

The central bunch is focused onto the entrance of the RFQ, while the neighboring bunches experience a net deflection over a period of two $\mathrm{rf}$ cycles (or $\Delta \mathrm{L}=$ $2 \beta \lambda=22 \mathrm{~mm}$ ). For a deflecting potential of \pm 3000 volts, the deflection angle for the neighboring bunches is equal to

$$
\theta_{\mathrm{def}}=18 \mathrm{mrad}
$$

Employing linear beam optics for a parallel beam with a final focus with focal length $f$, a deflected beam with a deflection angle of $\theta_{\mathrm{def}}$ would experience a transverse offset of

$$
\Delta r=f \times \theta_{\text {def }}
$$

at the focus location. For a final focal length of $\mathrm{f}=350 \mathrm{~mm}$, the transverse offset at the RFQ entrance for a deflecting potential of \pm 3000 volts is

$$
\Delta r \sim 6 \mathrm{~mm} .
$$

The transverse acceptance at the RFQ entrance is about $\pm 3 \mathrm{~mm}$, and so this offset is sufficient to block out the neighboring bunches.

A precise description of the deflection plates was initiated in an effort to better understand and improve on the actual performance of the fast chopper. Detailed beam dynamics simulations of the fast chopper system were performed using the General Particle Tracer (GPT) [30] beam dynamics package, a time-based multiparticle code with fifth order Runga-Kutta integration. The simulation package includes hooks for user written routines for accelerating elements and beam dynamics routines. A detailed code for RFQ, developed by our group and reported in Ref. [31], was incorporated into the beam dynamics simulations. The RFQ fields were obtained using the eight-term potential approach with coefficients determined with a least $\chi^{2}$ fit to the electrode surfaces [32], and also using a precise three-dimensional treatment [33] with fields calculated with the CST code [34]. A routine for the deflection plates was also incorporated, including the fast switching of the electric fields. A more detailed discussion of the fast chopper simulations for single bunch selection can be found in Ref. [29].

An accurate map of the electric field of the deflection plates was also necessary. Figure 4(a) displays a photograph of the deflection plates with the upstream and downstream flanges removed, showing the cross section configuration 

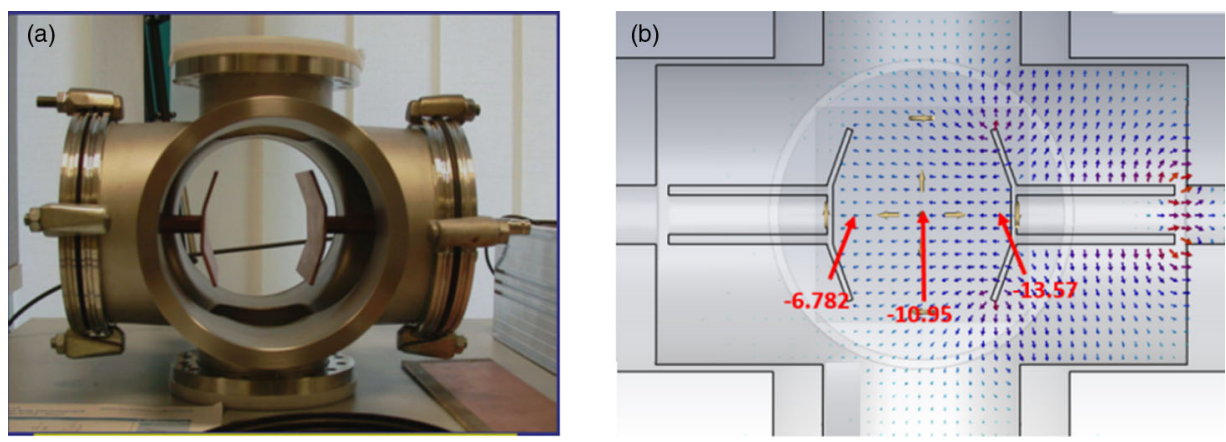

FIG. 4. Fast chopper deflection plates. (a) Photo of vacuum tank showing the deflection plates: leftmost plate is grounded, rightmost plate is connected via feedthrough to HV. (b) Simulation of the electric field between deflection plates. Numbers in red ( $\mathrm{E}_{\text {field }}$ in $\mathrm{kV} / \mathrm{m}$ ) indicate a variation of factor of 2 due to field asymmetry.

within the vacuum tank. The rightmost deflection plate is connected via a feedthrough to the HV source, whereas the leftmost plate is grounded to the vacuum tank. A study based on 3D calculations of the electric field was made with the CST microwave studio computer simulation code [34], where exact dimensions of the deflection plates and vacuum vessel were used as the input. The simulations show that the inherent asymmetry in the deflection plates results in an asymmetric electric field [Fig. 4(b)]. A new design of the deflection plates is currently under way to minimize the electric field asymmetry and provide for more effective single bunch selection. The calculated electric field map for the existing deflection plates was used as input to the beam dynamics simulations of the fast chopper.

The beam dynamics simulations involved transporting a dc beam through the LEBT, positive-negative deflection between solenoids \#2 and \#3, and a final focus onto the entrance of the RFQ. The values of solenoid magnetic fields and deflection plates electric fields used in the simulations were very close to values experimentally obtained for "single bunch selection" as described in Sec. III. The results of the simulations are very similar to the experimental results. Simulated single bunch transmission was obtained with about 50\% transmission. Figure 5 shows a snapshot in time for the moment the beam impinges on the entrance of the RFQ, for particles on that survived the upstream collimators. The figure shows a scatter plot of the longitudinal verses the transverse location of the particles. Grey particles are those that were eventually not transmitted through the RFQ. The light blue particles are particles from the central bunch, and the red and dark blue particles are those in the previous and next bunches that were transmitted, amounting to $20 \%$ of the total transmitted particles for this simulation. A new design of the deflection plates is under way to increase the transmission of the central bunch and to lower as much as possible the neighboring bunches. Further details on the fast chopper simulations will be discussed elsewhere [35,34].

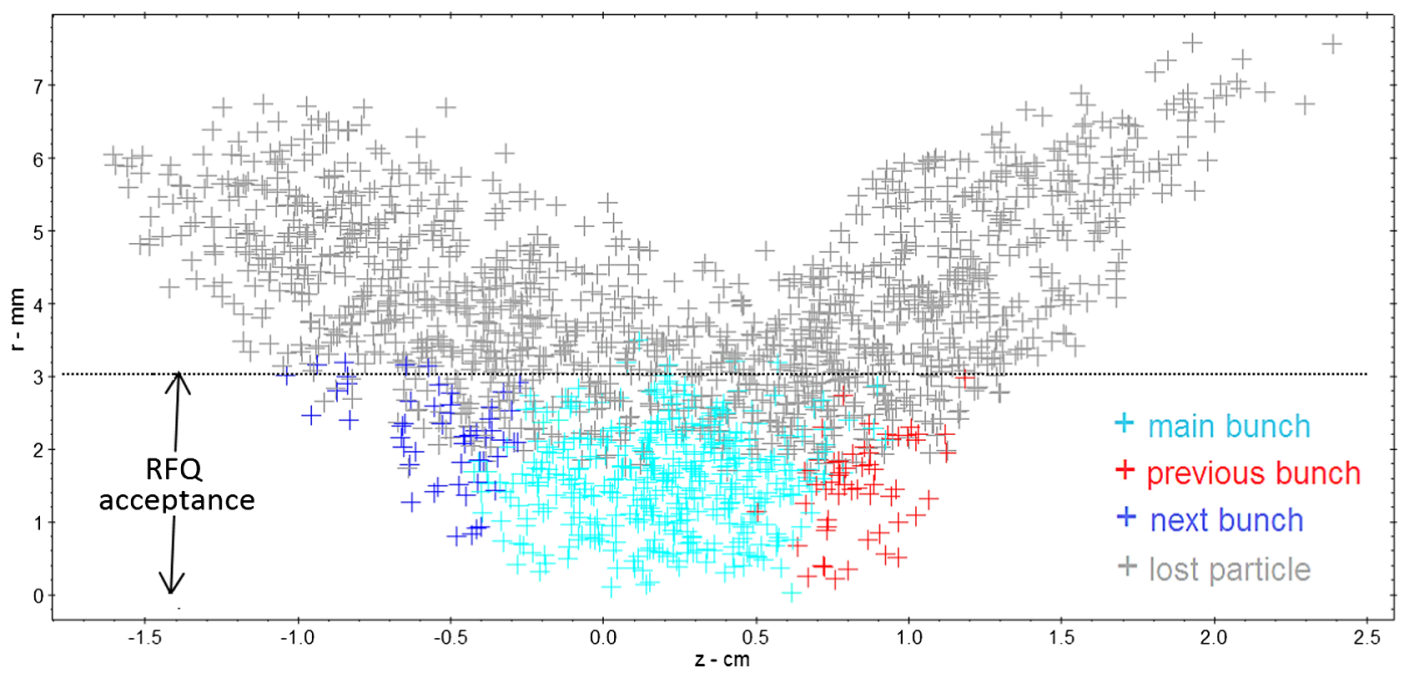

FIG. 5. Beam dynamics simulations: Snapshot of chopped beam at entrance to RFQ. colored particles are transmitted by RFQ-light blue particle eventually became main bunch, red and dark blue eventually became previous and next bunch—grey particles are not transmitted. 


\section{IMPLEMENTATION OF THE FAST CHOPPER SYSTEM FOR SINGLE RFQ BUNCH SELECTION}

The SARAF RFQ, at rf frequency of $176 \mathrm{MHz}$, produces protons or deuterons bunches at $5.6 \mathrm{~ns}$ intervals with temporal width of $0.3 \mathrm{~ns}$ FWHM per bunch. The ability to select a single RFQ bunch will enable production of sharp neutron pulses for precise TOF measurements of neutron induced reactions. This capability would be especially beneficial for SARAF phase II, which is expected to provide unprecedented flux for fast neutrons at energies of 1-20 MeV [20].

A scheme for single bunch selection was successfully developed with the existing slow chopper deflection electrodes situated before the RFQ. The idea involves modifying the $\mathrm{HV}$ electronics to include both positive and negative deflection, provided by positive and negative $\mathrm{HV}$ supplies. A fast HV switch reverses deflection polarity, and is triggered when single RFQ prebunch transmission is desired. The polarity switch occurs in a time scale of 20-30 ns, depending on capacitive load and impedance. Although there is no section of the dc beam that does not experience deflection, an unhindered prebunch nevertheless traverses to the entrance of the RFQ following equal amounts of positive and negative deflection. This technique relies on exact cancellation between positive and negative deflection, and depends strongly on symmetric fields and switch between the alternating polarities.

The desired specifications for a fast chopper for phase I of SARAF are for single bunch selection optimized for deuterons at a repetition rate of up to $200 \mathrm{kHz}$. The estimates for deflection voltages for deuterons at $20 \mathrm{keV} / \mathrm{u}$ are for $\pm 5 \mathrm{kV}$ on the deflection electrodes, with a contingency margin bringing the $\mathrm{HV}$ to $\pm 7 \mathrm{kV}$. Reversing $\mathrm{HV}$ polarity on the deflection plates involves charging of the deflection plate capacitance, where the $\Delta \mathrm{V}$ is expected to be $10 \mathrm{kV}$, with a possible maximum of $14 \mathrm{kV}$. The total capacitive load
$\left(\mathrm{C}_{L}\right)$ is estimated at $100 \mathrm{pF}$, and includes the capacitance of the deflection plates, HV switch, and cables. The design is for HV polarity switch within a $\Delta \mathrm{t}$ of 20-30 nanoseconds. The design criteria are therefore for a maximum instantaneous switch current of about 50 amperes, and a total power load of under $2 \mathrm{~kW}$. Behlke Electronics GmbH [36] offers HV switches with push-pull circuitry consisting of two identical MOSFET switching paths controlled by a common driver, specially designed for precise $\mathrm{HV}$ switching. We opted for model number HTS 161-06-GSM with oil immersion cooling and with the high switching frequency option, which suits our application favorably. The HV supplies chosen were Spellman PTV10P350 and PTV10N350 [37] for the positive and negative voltages, respectively, each capable of providing up to $350 \mathrm{~mA}$ current at $10 \mathrm{kV}$. The $\mathrm{HV}$ supplies are connected to the HV switch via the series resistor $\mathbf{R}_{S}$, which must be of low inductance and high power rating to provide impedance matching and limit the instantaneous switch currents. Kanthal slab resistors [38] with $100 \Omega$ resistance and with forced air cooling were chosen, with $\mathrm{HV}$ buffer capacitors of $100 \mathrm{nF}\left(\mathrm{C}_{B}\right)$ to maintain a steady voltage. Short line paths and very low inductance components are essential to prevent fly-back voltages and ensure fast discharge. The operation of the Behlke HV switch and the Spellman HV supplies are all computer controlled and are operated remotely. A schematic diagram of the HV switching circuit is shown in Fig. 6.

The trigger for single bunch selection must be taken from the main SARAF rf oscillator to enable effective single prebunch transmission at the desired rf phase. The single bunch repetition rate is obtained by scaling down the SARAF rf frequency of $176 \mathrm{MHz}$ to the desired usable repetition rate (up to $200 \mathrm{kHz}$ ). This is done with nuclear instrumentation module standard (NIM) based electronics which downscales and trigger on the main rf signal, while maintaining the original rf phase. A transistor-transistor logic signal levels (TTL) trigger signal is generated in the format required by the Behlke HV switch, with provision to
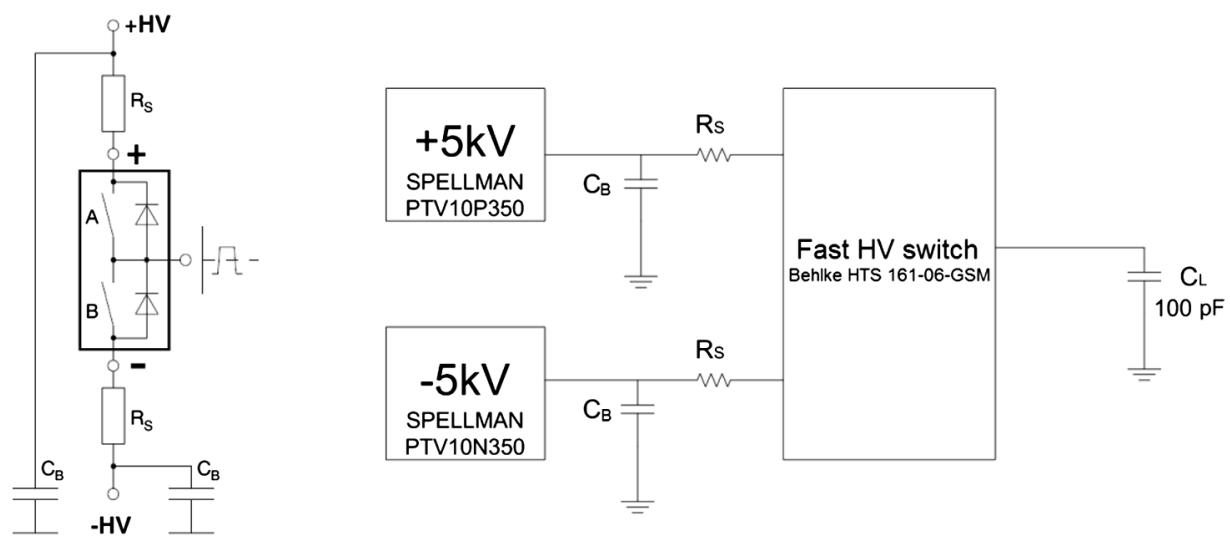

FIG. 6. Schematic diagram of $\pm \mathrm{HV}$ for the deflection electrodes. The left side shows basic schematic diagram of push-pull HV switch. The right side shows the circuit implemented for the deflecting electrodes to enable fast chopper operation, where the capacitive load $\mathrm{C}_{L}$ is provided by the deflection electrodes. 

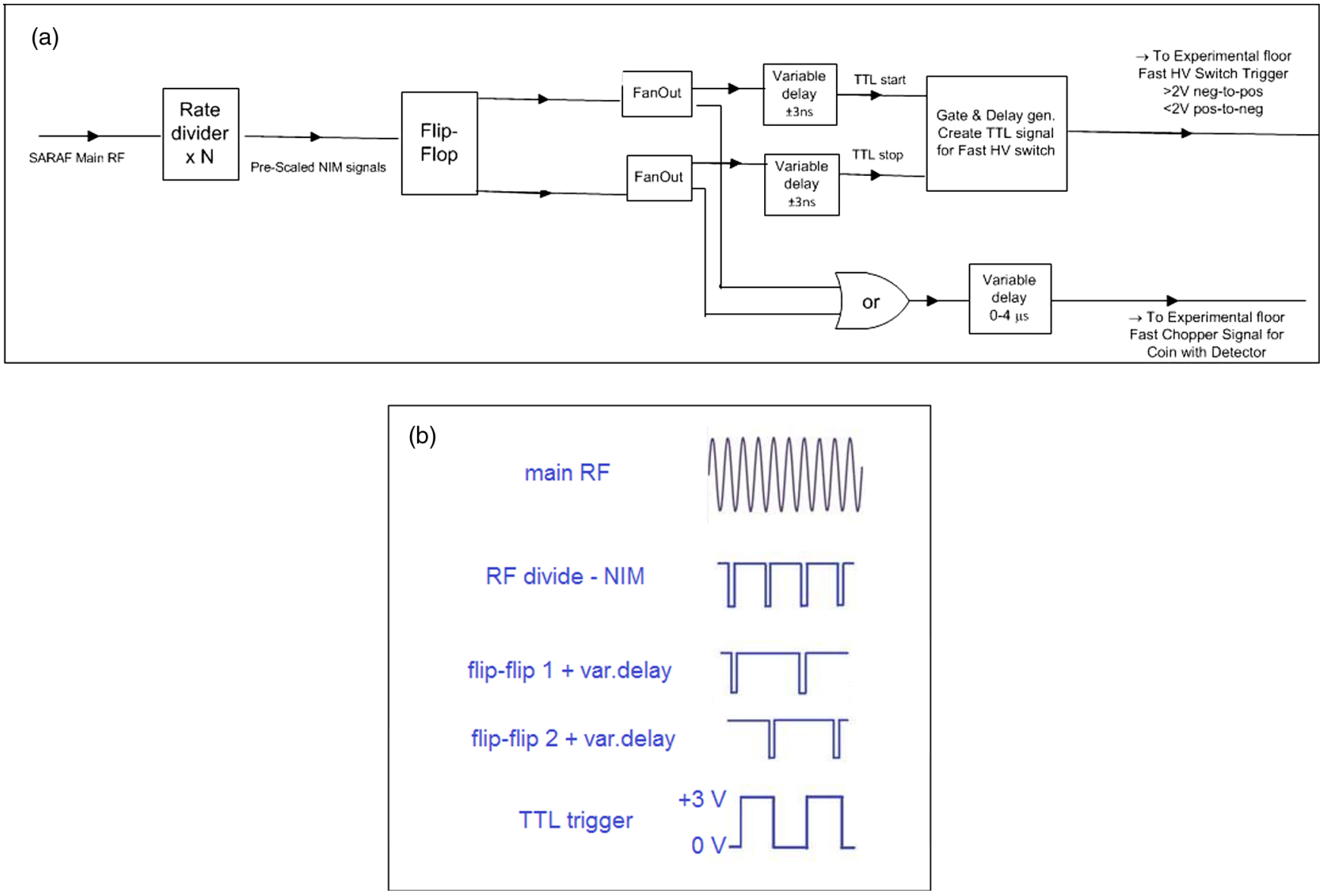

FIG. 7. (a) Electronic logic diagram of trigger for the fast chopper HV switch. (b) The trigger involves a down scale of the main SARAF rf to the desired single bunch repetition rate, separate variable delays for the negative-to-positive and positive-to-negative HV swings, and formation of appropriate TTL pulse required for the Behkle HV switch.

adjust the phase of the timing of the trigger to coincide with the phase of the RFQ. A HV swing from negative to positive HV occurs when the TTL trigger signal goes above 2 volts, and a positive to negative HV swing occurs when the TTL signal goes below 2 volts. Both turn-on and turnoff circuits require each their own delay for precise phasing with the RFQ bunch phase (otherwise rf frequency beating may occur for a downscale frequency that is not an exact multiple of the original). The fast chopper trigger (after appropriate delay) also serves as the TOF start for the TOF measurements. The NIM based electronics logic for the fast chopper trigger is shown in Fig. 7.

To accommodate positive and negative HV deflection, a redesigned water-cooled beam blocker with a $50 \mathrm{~mm}$ aperture in the middle for transmission of the nondeflected beam was designed and installed (Fig. 2). Also, an additional $12 \mathrm{~mm}$ aperture just upstream of the entrance to the RFQ was installed. Fast HV switching requires careful attention to circuit design to avoid overshoots due to stray inductance, or oscillations due to impedance mismatching. Figure 8(a) shows an oscilloscope trace of the HV profile applied on the deflection plates, with a $30 \mathrm{~ns}$ HV switch from -3.25 to $+2.75 \mathrm{kV}$ using the Behlke fast push-pull HV switch. Care has been taken to minimize overshoot and ringing, with good results but still room for improvement. Provisions for accelerator safety include a field probe at the location of deflection plates that triggers fast switching off of the ion source in the event of loss of deflection field. Figure 8(b) shows the signals induced on the BPM situated in the MEBT, and the FFC in the D-plate, demonstrating nearly single bunch selection for the HV profile shown in Fig. 6(a).

Effective single RFQ bunch selection requires a constant nonfluctuating proton or deuteron beam from a steady ion source. Initial adjustment of the LEBT focusing solenoids, mostly solenoid 2 and solenoid 3, along with fine-tuning of the magnetic steerers, was found to be necessary for obtaining optimum single bunch selection. The proton or deuteron single bunches exiting the RFQ were measured by induced pulse on the BPM detectors in the MEBT, where an approaching bunch draws a positive current, and a receding bunch draws negative current. The most optimum conditions we are able to obtain for single bunch selection for protons was for positive $2.75 \mathrm{kV}$ and negative $3.25 \mathrm{kV}$, 

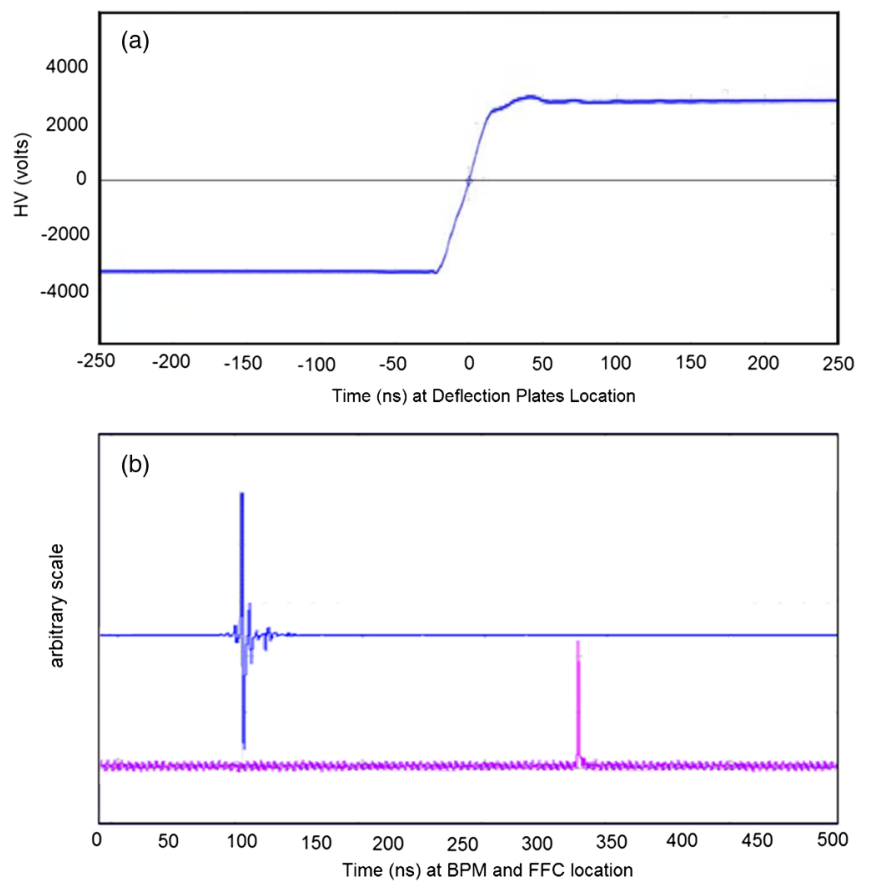

FIG. 8. (a) Oscilloscope trace of HV applied to deflector plates. The fast chopper trigger initiates a $30 \mathrm{~ns} \mathrm{HV}$ switch from -3.25 to $+2.75 \mathrm{kV}$. (b) Detectors response following fast chopper trigger. The blue trace is signal induced on the BPM situated in the MEBT, and the purple trace is beam induced signal on the FFC situated in the D-plate (averaged over four shots).

with fast switching of $28 \mathrm{~ns}$ duration. The asymmetry of the optimum HV configuration is attributed to the inherent asymmetry of the deflection plates, namely that the beamright deflection plate is connected to the negative-positive $\mathrm{HV}$, while the beam-left electrode is grounded and at the same potential as the conductive vacuum tank.

Figure 9 shows the BPM traces on a fast $10 \mathrm{GHz}$ sampling oscilloscope for 50 consecutive triggers for single RFQ bunch selection. Figure 9 indicates that there

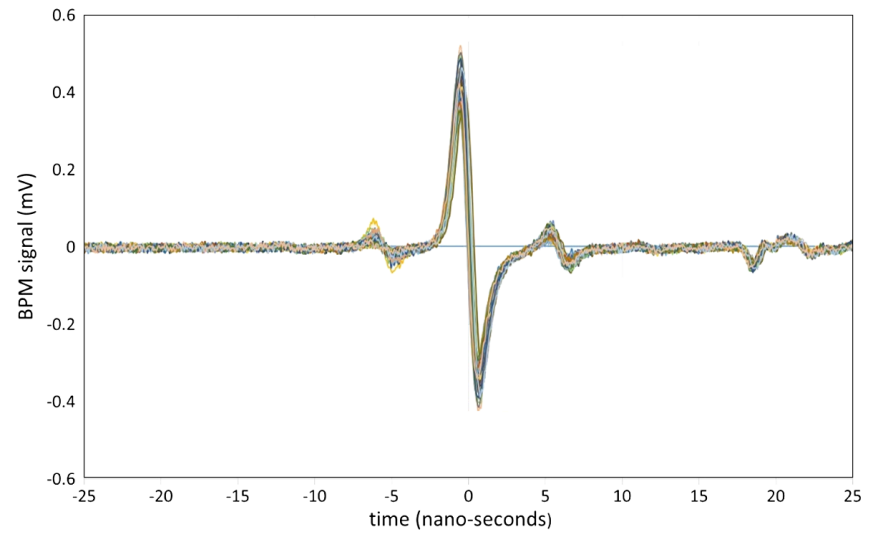

FIG. 9. Superposition of waveforms of signals induced on the beam position Monitor by 50 consecutive fast chopper triggers. Induced signals indicate effective single RFQ bunch selection with less than $15 \%$ satellite bunches. is good uniformity over the 50 triggers. For our most optimal quasisingle bunch selection, we were not able to eliminate the satellite bunches, one before and one after each main bunch, together corresponding to about $15 \%$ of the total beam. The single bunch transmission, measured compared to cw beam, was about $40 \%$, as measured by the amplitude of the induced signal on the BPM detector. Higher pulse transmission can be obtained, even above $50 \%$, but at the cost of higher transmission for the satellite bunches. It is anticipated that better filtering design of the HV switching circuit, along with more systematic tune for a symmetric beam, will decrease the amount of satellite peaks and increase transmission of the single bunch. Simulations show that a more symmetric deflection characteristics will significantly improve the single bunch characteristics for triggers corresponding to the reverse HV polarity switch.

\section{NEUTRON TIME-OF-FLIGHT MEASUREMENTS WITH FAST CHOPPER SYSTEM AT SARAF}

A time-of-flight experiment was set up to demonstrate the capabilities of the fast chopper system and to test the timing resolution achievable. A thick LiF target (full proton stopping) was placed in the target position in the D-Plate, about 2 meters downstream of the PSM. A liquid scintillator detector (NE315 St. Gobain 2.5" × 2.5" [39]) situated inside a borated polyethylene shield was placed about 8 meters downstream of the LiF target. The detector photomultiplier (PMT) anode signal was fed to a linear fan-in-fan-out, where one of the output signals was fed to a fast discriminator to establish coincidence with the fast chopper trigger. The second output signal was fed to a two-channel 1.6 GHz 12-bit Keysight [40] M9703A waveform digitizer card [40]. The signal from the BPM was fed to the second channel of the waveform digitizer card to serve as a precise reference for TOF start and also to monitor the quality of the single bunch selection on an event by event basis, with the possibility of eliminating events with undesirable single bunch characteristics. The signal from the liquid scintillator served as the TOF stop and also made available the possibility of pulse height analysis and neutron-gamma pulse-shape discrimination (PSD). The trigger to the waveform digitizer was formed by the coincidence between the fast chopper trigger and the discriminated liquid scintillator signal, where the fast chopper trigger signal was time defining, and the liquid scintillator logic signal sufficiently broad to cover the required TOF range. The logic signal for the liquid scintillator detector was widened to $1.2 \mu \mathrm{s}$ to allow for the full desired TOF interval. The signal for the fast chopper trigger, originating at the control room, was fed to a gate and delay generator where the timing delay was varied at the control room to allow for on-line establishing of the optimum timing, i.e., maximum coincidence and 


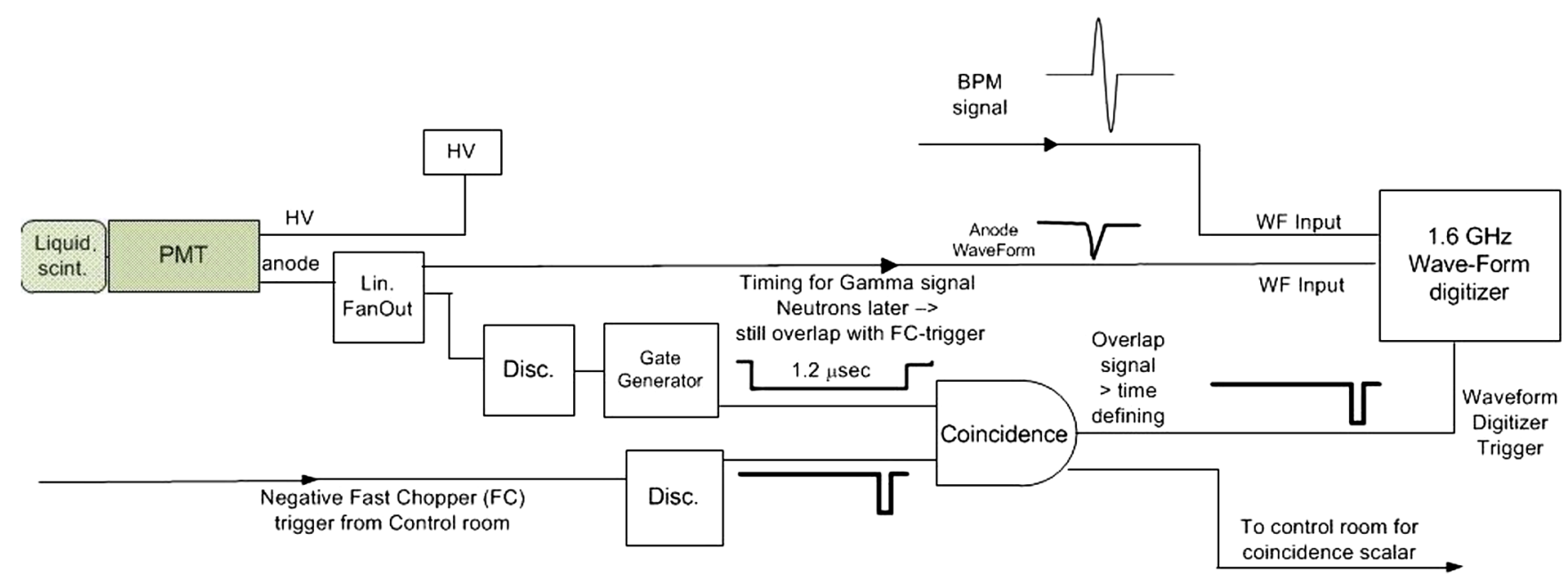

FIG. 10. Fast timing diagram for TOF via waveform measurements of the liquid scintillator detector and the BPM with the fast chopper (FC) serving as a trigger for the waveform digitizer.

gamma neutron time of flight within the desired waveform range. A schematic of the electronics diagram for data acquisition is shown in Fig. 10.

Figure 11 shows typical signals obtained with the waveform digitizer: Fig. 11(a) shows a BPM signal, 11(b) shows a detector signal for a gamma candidate, and 11(c) shows a detector signal for a neutron candidate. Linear interpolation was performed to obtain a fast algorithm but with precise timing analysis. For the BPM signal, a linear interpolation was made for the inflection point of the positive-negative signal crossing of the $x$ axis [see the blue dot in Fig. 9(a)], where the zero of the $\mathrm{x}$ axis was determined by a linear fit of the background. For the detector signal, a linear interpolation was made for the rise of the scintillator signal between $25 \%$ and $75 \%$ of maximum signal height, where the zero crossing with the $\mathrm{x}$ axis determined the timing, as characterized by the blue dots in Figs. 9(b) and 9(c).

The waveforms shown in Figs. 11(b) and 11(c) are qualitatively different for gammas and for neutrons due to the special characteristics of the liquid scintillator, thereby enabling pulse shape discrimination on an event by event basis [41]. Neutron-gamma pulse shape discrimination was performed by defining a quantifier that compares the tail of a given pulse with the total charge of the pulse. The PSD quantifier is given by

$$
P S D=\frac{\sum_{i_{\max }+16 \mathrm{~ns}}^{i_{\max }+125 \mathrm{~ns}} c h_{i}}{\sum_{i_{\max }-10 \mathrm{~ns}}^{i_{\max }+125 \mathrm{~ns}} c h_{i}},
$$

where $i_{\max }$ represents the channel for which the pulse height is maximum. Parameters of the PSD quantifier were adjusted off-line to obtain the optimal neutron-gamma PSD. Excellent neutron-gamma PSD was realized despite internal PMT and base reflections which we were not able to totally eliminate.

The resulting TOF spectra are shown in Fig. 12 for protons at $3.5 \mathrm{MeV}$ bombarding a thick $\mathrm{LiF}$ target, with the liquid scintillator situated at the end of beam line- 0 at
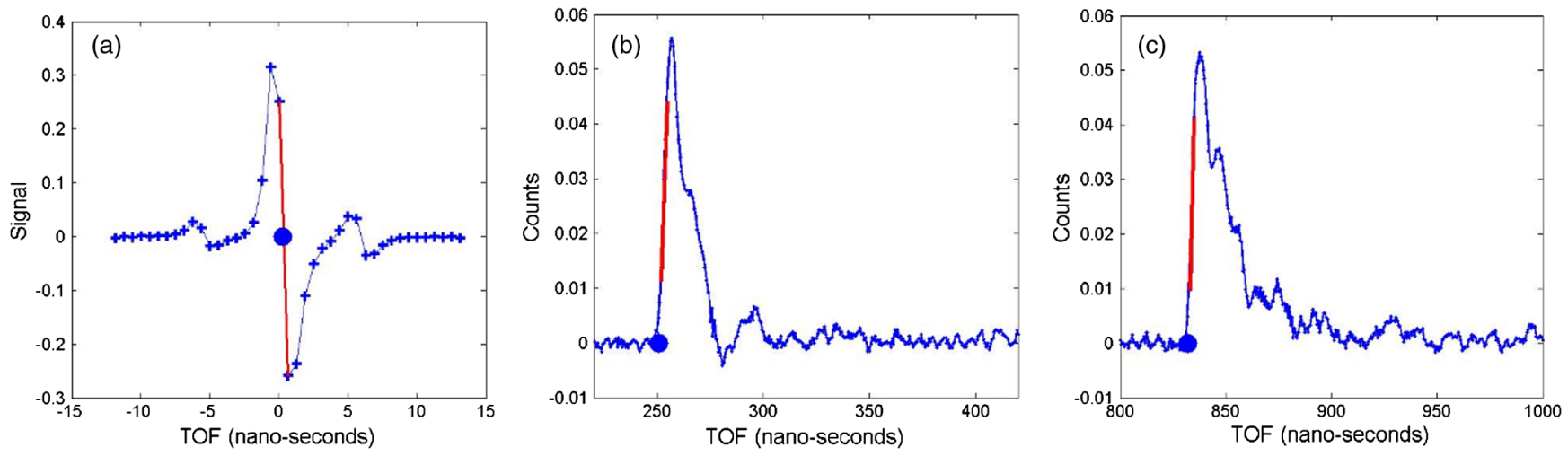

FIG. 11. Waveforms obtained with the $1.6 \mathrm{GHz}$ waveform digitizer: (a) typical BPM waveform, with satellite peaks clearly visible, (b) detector signal for a gamma candidate, (c) detector signal for a neutron candidate. Red lines show the linear fit and the large blue dot the timing obtained by zero crossing with the $\mathrm{x}$ axis. 

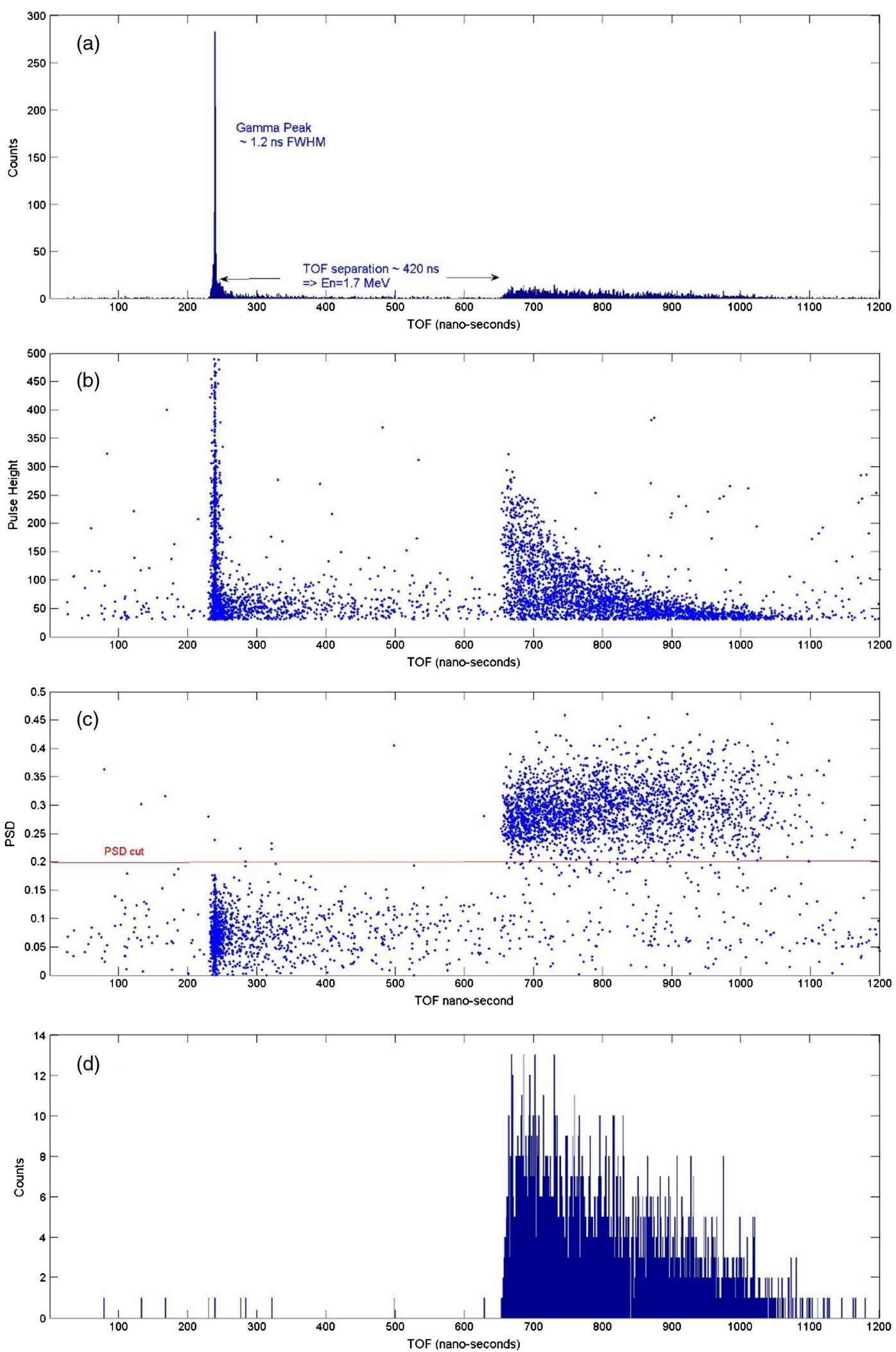

FIG. 12. TOF spectra for neutrons produced in the $\mathrm{p}+\mathrm{LiF}$ thick target reaction at $\mathrm{E}_{p}=3.5 \mathrm{MeV}$ and at $0^{\circ}$. (a) TOF spectrum, indicating a gamma peak with $\Delta \mathrm{t}=1.2 \mathrm{~ns}$. (b) TOF vs pulse height ( $E_{\text {threshold }}^{\text {neutron }} \sim 0.3 \mathrm{MeV}$ ). (c) TOF vs PSD where the red line represents PSD cut. (d) TOF spectrum for events that pass the PSD cut, indicating neutrons created in the $\mathrm{p}+\mathrm{Li}$ reactions. 
$0^{\circ}$ and 8 meters downstream of the LiF target. Figure 12(a) shows the total TOF spectrum for events with pulse height $>30 \mathrm{mV}$ (corresponding to $\mathrm{E}_{n}>300 \mathrm{keV}$ ). The TOF resolution obtained for the gamma peak is $\Delta \mathrm{t}=1.2$ nanoseconds FWHM, consistent with the Nyquist limit [42] for a $1.6 \mathrm{GHz}$ sampling rate. The time of the fastest neutrons is consistent with neutron energy of $\mathrm{E}_{n}=1.7 \mathrm{MeV}$, as dictated by the ${ }^{7} \mathrm{Li}(\mathrm{p}, \mathrm{n})^{7} \mathrm{Be}$ reaction kinematics. Figure $12(\mathrm{~b})$ shows a scatter plot of TOF vs pulse height, where, again, the maximum pulse height is consistent with neutron at $\mathrm{E}_{n}=1.7 \mathrm{MeV}$. Figure 12(c) shows a scatter plot of TOF vs the PSD quantifier, where the horizontal red line shows where the PSD cut was made. Figure 12(d) shows the total TOF spectrum for events that passed the PSD cut, and it is clearly observed that only neutrons survived the PSD cuts. The few events with TOF not identified with neutrons are primarily anomalous events such as double events within the coincidence gate, etc. Only about $3 \%$ of the neutrons did not pass the PSD cut.

\section{SUMMARY AND PLANS FOR FUTURE DEVELOPMENT}

This paper reports on progress with a fast chopper at the SARAF accelerator to provide for single RFQ bunch selection. The fast chopper system makes use of the existing slow chopper deflection plates situated in the LEBT. A bipolar electric field is applied on the deflector plates where triggering of a fast HV switch initiated a fast polarity switch enabling a single prebunch to enter the RFQ. The fast chopper system was successfully implemented with encouraging single bunch selection characteristics, including single bunch transmission approaching $50 \%$ and with satellite bunch contamination of under $15 \%$, with a single bunch repetition rate approaching $200 \mathrm{kHz}$.

Several modifications of the deflection plates are being implemented, where dedicated beam dynamics simulations show that significant improvement in single bunch selection can be achieved with a more uniform deflection electric field. The issue of space charge neutralization and deneutralization is of significance for chopping of high current beams in the LEBT section, and techniques for deneutralization of the chopping section are being considered. The implementation of fast switching of bipolar deflection fields was made possible by recent advances in fast HV switching technology. Continued advances of this technology will make possible further improvements in single bunch selection characteristics and increased repetition rate.

A TOF system with a liquid scintillation detector has been set up to test the timing characteristics available with the fast chopper system. Excellent TOF properties have been obtained, with sharp timing resolution with $\Delta \mathrm{t} \sim$ $1.2 \mathrm{~ns}$ (consistent with the Nyquist limit for the $1.6 \mathrm{GHz}$ waveform digitizer used) and with good gamma-neutron pulse shape discrimination. The gamma peak is almost devoid of satellite peaks, suggesting larger transmission loss in the PSM for the satellite bunches as compared to the central bunch. Simultaneous measurements of the TOF along with the response of the BPM enables off-line selection of events with good single bunch characteristics on an event by event basis.

Single RFQ bunch selection by means of the fast chopper being developed at SARAF provides TOF capabilities for measurements of nuclear reactions that include nuclear spectroscopy, study of fission fragments and prompt fission neutron energy distributions, and various aspects of nuclear astrophysics. In addition, neutron TOF techniques can be very useful for security applications, luggage or container interrogation, and other industrial applications where neutron TOF can provide information on elemental composition of suspected items. Neutron TOF capabilities, especially at phase II of SARAF, will provide exceptional opportunities for neutron induced reaction measurements including fission and $(\mathrm{n}, \mathrm{Xn})$ reactions important for nuclear technology as well as fundamental research.

\section{ACKNOWLEDGMENTS}

We would like to thank the SARAF accelerator operation staff for delivering a dedicated ion beam. We also thank the FP7-SPIRAL2PP project for the design, construction and test of the slow chopper that was used in this study. This project was partially supported by the Israeli Atomic Energy Commission (IAEC) and the European Commission-Joint Research Centre (EC-JRC) joint project initiative and the Israel Ministry of Energy, Research Grant No. 217-11-023.

[1] Neutron Scattering, edited by K. Sköld and D. L. Price (Academic Press, New York, 1986).

[2] F. Caspers, Review of fast beam chopping, in XXII International Linear Accelerator Conference Linac'04, Lübeck, Germany (2004), pp. 578-582, http://cds.cern.ch/ record/804997/files/ab-2004-096.pdf.

[3] M. A. Clarke-Gayther, A two stage fast beam chopper for next generation high proton drivers, in Proceedings of the 2nd International Particle Accelerator Conference, San Sebastián, Spain (EPS-AG, Spain, 2011), pp. 1786-1788, http://accelconf.web.cern.ch/accelconf/ipac2011/papers/ tups 104.pdf.

[4] A. Caruso et al., Experimental performance of the chopper for the ESS LINAC, https://accelconf.web.cern.ch/ AccelConf/ipac2018/, pp. 1709-1711.

[5] A. Aleksandrov and C. Deibele, Experimental study of the SNS MEBT chopper performance, in Proceedings of the International Particle Accelerator Conference, Kyoto, Japan (ICR, Kyoto, 2010) pp. 831-833.

[6] T. Kato et al., Beam study with RF choppers in the MEBT of the J Parc proton linac, in Proceedings of the 2003 Particle Accelerator Conference, Portland, OR (IEEE, New York, 2003), pp. 1455-1457. 
[7] F. Caspers et al., The CERN-SPL chopper concept and final layout, in Proceedings of the 9th European Particle Accelerator Conference, Lucerne, 2004 (EPS-AG, Lucerne, 2004), pp. 1144-1146, http:// accelconf.web.cern.ch/AccelConf/e04/PAPERS/TUPLT007 .PDF.

[8] C. Wiesner, M. Droba, O. Meusel, D. Noll, O. Payir, U. Ratzinger, and P. Schneider, Experimental performance of an $E \times B$ chopper system, Phys. Rev. Accel. Beams 20, 020101 (2017).

[9] K. W. Leo, T. Adachi, and K. Takayama, Einzel lens chopper and behavior of the chopped beam in the KEK digital accelerator, Phys. Rev. ST Accel. Beams 16, 043502 (2013).

[10] G. Le Dem and M. Di Giacomo, A single bunch selector for the next low $\beta$, in Proceedings of the 16th IEEE International Pulsed Power Conference, Albuquerque, NM (IEEE, Piscataway, NJ, 2007) pp. 158-160, http://hal .in2p3.fr/in2p3-00169268/document.

[11] X. Ledoux et al. (NFS collaboration), Spiral-2 for neutron production, in the 13th international topical meeting on nuclear applications of accelerators, AccApp '17, Quebec (2017), http://accapp17.org/wp-content/uploads/2017/09/ AccApp2017_Ledoux_web.pdf.

[12] X. Ledoux, The neutrons for science facility at SPIRAL-2, Radiation Protection Dosimetry 180, 115 (2018).

[13] F. Consoli, P. Balleyguier, and M. D. Giacomo, Broadband electromagnetic characterization of a $100 \Omega$ travelingwave electrode by measuring scattering parameters, Phys. Rev. ST Accel. Beams 16, 072001 (2013).

[14] R. Madrack and D. Wildman, A fast chopper for medium energy beams, J. Inst. 9, T10009 (2014).

[15] S. Nath, R. R. Stevens, Jr., and T. P. Wangler, Conceptual designs of beam choppers for RFQ linacs, in Proceedings of the Particle Accelerator Conference, Dallas, TX, 1995 (IEEE, New York, 1995) pp. 1846-1848, https:// accelconf.web.cern.ch/accelconf/p95/ARTICLES/WAQ/ WAQ02.PDF.

[16] L. Prost, J. P. Carneiro, and A. Shemyakin, Low emittance growth in a LEBT with un-neutralized section, in Proceedings of the 7th International Particle Accelerator Conference IPAC'16, Busan, Korea (Pohang Accelerator Laboratory, Pohang, Korea, 2016), pp. 1317-1319, http:// accelconf.web.cern.ch/accelconf/ipac2016/papers/tupmr033 .pdf.

[17] R. Laxdal, M. Pasini, and L. Root, Beam dynamics design study and beam commissioning of the ISAC two frequency chopper', in Proceedings of 21st International Linear Accelerator Conference LINAC'02, Gyeongju, Korea (Pohang Accelerator Laboratory, Pohang, Korea, 2002), pp. 409-411, http://accelconf.web.cern.ch/accelconf/102/PAPERS/TU441 .PDF.

[18] V. Dangendorf et al., FNeuRad proposal, 2007, http:// fneurad.de/intro.html.

[19] R. W. Hamm et al., A single pulse sub-nanosecond proton RFQ, in The Tenth international topical meeting on nuclear applications of accelerators, AccApp '11, Knoxville, TN, 2011, Report No. LLNL-CONF-477421, https://e-reports-ext .llnl.gov/pdf/481249.pdf.
[20] I. Mardor et al., The Soreq Applied Research Accelerator Facility (SARAF): Overview, research programs and future plans, Eur. Phys. J. A 54, 91 (2018).

[21] A. Kreisel et al., Phase-I proton/deuteron linac beam operation status, in Proceedings of the 27th Linear Accelerator Conference, LINAC2014, Geneva, Switzerland (2014), pp. 779-774, http://accelconf.web.cern.ch/AccelConf/ linac2014/papers/weiob02.pdf.

[22] L. Weissman et al., Installation, high-power conditioning and beam commissioning of the upgraded SARAF 4-rods RFQ, J. Instrum. 13, T05004 (2018).

[23] N. Colonna, F. Gunsing, and F. Kappeler, Neutron physics with accelerators, Prog. Part. Nucl. Phys. 101, 177 (2018).

[24] H. Harano, T. Matsumoto, Y. Tanimura, Y. Shikaze, M. Baba, and T. Nakamura, Monoenergetic and quasimonoenergetic neutron reference fields in Japan, Radiation Measurements 45, 1076 (2010).

[25] J. L. Szabo and J. L. Boutaine, Some examples of industrial uses of neutron sources, Radiation Protection Dosimetry 70, 193 (1997).

[26] N. Pichoff et al., The SARAF-linac project 2018 status, in Proceedings of the 9th International Particle Accelerator Conference IPAC2018, Vancouver BC, Canada (2018), pp. 994-996, http://accelconf.web.cern.ch/AccelConf/ ipac2018/papers/tupak015.pdf.

[27] A. Shor et al., Performance of beam chopper at SARFAF via RF deflector before the RFQ, in Proceedings of XXVI Linear Accelerator Conference LINAC2012, Tel-Aviv, Israel (2012), pp. 1038-1040, http://accelconf.web.cern.ch/ accelconf/linac2012/papers/thpb094.pdf.

[28] A. Caruso et al., Preliminary design of the slow chopper for the SPIRAl 2 project, in Proceedings of XXIV Linear Accelerator Conference LINAC'08, Victoria, BC, Canada (2009), pp. 891-893, http://accelconf.web.cern.ch/ accelconf/LINAC08/papers/thp046.pdf.

[29] A. Shor et al., Fast beam chopper at SARAF accelerator via RF deflector before RFQ, J. Instrum. 7, C06003 (2012).

[30] GPT-General Particle Tracer code, Pulsar Physics, Burghstraat 475614 BC, Eindhoven, The Netherlands, http:// www.pulsar.nl/gpt/.

[31] B. Bazak et al., Simulations of ion beam loss in RF linacs with emphasis on tails of particle distributions, in Proceedings of the XXVI Linear Accelerator Conference LINAC'10, Tsukuba, Japan (2016), pp. 956-958, https://accelconf .web.cern.ch/accelconf/LINAC2010/papers/thp091.pdf.

[32] B. Bazak, Simulations of ion beam loss in RF linacs with emphasis on tails of particle distributions, M.Sc. thesis, Hebrew University of Jerusalem 2008 and Soreq NRC, Report No. SNRC-4130, 2009.

[33] B. Mustapha, A. A. Kolomiets, and P. N. Ostroumov, Full three-dimensional approach to the design and simulation of a radio-frequency quadrupole, Phys. Rev. ST Accel. Beams 16, 120101 (2013).

[34] CST-Computer Simulation Technology GmbH, Bad Nauheimer Strasse 1964289 Darmstadt, Germany, http://www .cst.com/.

[35] A. Shor et al., Simulations of fast chopper for RFQ single bunch selection (to be published).

[36] Behlke Power Electronics GmbH Am Auernberg 4, 61476 Kronberg, Germany, http://www.behlke.com/. 
[37] Spellman High Voltage Electronics, 475 Wireless Blvd., Hauppauge, NY, http://www.spellmanhv.com/.

[38] Kanthal slab resistors, Sandvik SE-734 27 Hallstahammar, Sweden, http://www.kanthal.com/.

[39] Saint-Gobain Cristaux, 104 Route de Larchant BP 521 77794 Nemours CEDEX, France, http://www.saint-gobain .com/.
[40] Keysight Technologies, 1400 Fountaingrove Parkway Santa Rosa, CA 95403-1738, http://www.keysight.com/.

[41] M. L. Roush, M. A. Wilson, and W. F. Hornyak, Pulse shape discrimination, Nucl. Instrum. Methods 31, 112 (1964).

[42] Ulf Grenander, Probability and Statistics: The Harald Cramér Volume (Wiley, New York, 1959). 